\title{
Measurement of $K_{\beta} / K_{\alpha} x$-ray intensity ratios and $K$ to $L$ shell total vacancy transfer probabilities for elements in the range $\mathbf{4 0} \leq \mathrm{Z} \leq \mathbf{5 0}$
}

\author{
A. Turşucu ${ }^{1 \star}$, P. Önder and D. Demir \\ Department of Physics, Faculty of Sciences, Ataturk University, 25240 Erzurum, Turkey.
}

Accepted 4 January, 2012

\begin{abstract}
The $K$ shell $\mathrm{x}$-ray intensity ratios $K_{\beta} / K_{\alpha}$ for 9 elements in the atomic range $40 \leq Z \leq 50$ have been determined for an excitation energy of $59.537 \mathrm{keV}$. The $K-L$ total vacancy transfer probabilities $\left(\eta_{K L}\right)$ for these elements have been determined, also. $K$ x-rays emitted by samples were detected using a high resolution $\mathrm{Si}(\mathrm{Li})$ semiconductor detector. The measured values were compared with the theoretical values calculated using Scofield's tables based on the Hartree-Slater and Hartree-Fock theories, and available experimental values. The agreement of measured values of intensity ratios and vacancy transfer probabilities were quite satisfactory with theoretical calculations.
\end{abstract}

Key words: $K$ shell intensity ratio, vacancy transfer probability, X-ray.

\section{INTRODUCTION}

Transition of an inner-shell electron from one shell to another will involve emission or absorption of high energy, short-wavelength radiation (x-rays). Usually, however, all of the energy levels of inner shells are occupied, so, in order to allow a transition to occur at all, a vacancy must be created in one of the inner shells. The energy required to do this can come from either the absorption of a sufficiently energetic photon, or from the impact with a high energy particle (Ewart, 2008).

The measurements of $K_{\beta} / K_{\alpha} x$-ray intensity ratios are significant for comparison with theoretical predictions based on atomic models in order to test the validity of these models. The $K_{\alpha} \mathrm{x}$-rays arise from transitions from the $L$ to the $K$ shell. The $K_{\beta}$ x-rays arise from transitions from the $\mathrm{M}-, \mathrm{N}-, \mathrm{O}$-, etc. to the $K$ shell. Atoms with vacancies in the inner shells are unstable and their stability can be regained by single or multiple electron transitions from the outer shells. When a single vacancy is created in an inner shell (for example, the $K$ shell), the vacancy is filled up by an electron coming from some higher shell. As a result, the vacancy is shifted to the

\footnotetext{
*Corresponding author. E-mail: ahmettursucu@atauni.edu.tr. Tel: + 904422314140 .
}

higher shell. The transfer coefficients, $\eta_{K L}$ describes the mean number of vacancies produced in the $L$ shell by one vacancy transfer probability and are important in many practical applications, such as nuclear electron capture, internal conversion of gamma rays, photoelectric effect, and atomic processes leading to the emission of $x$ rays, Auger electrons and computations for medical physics and irradiational process. X-ray fluorescence parameters such as intensity ratio and vacancy transfer probability are very important in understanding the ionization of atoms as well as for non-destructive elemental analysis in several fields such as material science, medical physics, industry and environmental science.

$K$ to $L$ shell vacancy transfer probabilities $\left(\eta_{K L}\right)$ of molybdenum (Mo), Palladium (Pd), and Cadmium (Cd) have been measured by repeatedly exciting them with the $K$ x-rays of Nickel (Ni) and Tin ( $\mathrm{Sn}$ ) induced by bremsstrahlung emanating from x-ray tube (Santra et al., 2005). Radiative vacancy transfer probabilities from the $L_{3}$ subshell to $\mathrm{M}$-, $\mathrm{N}$ - and $\mathrm{O}$ - shells and subshells in the atomic range $72 \leq Z \leq 92$ have been obtained (Tuzluca et al., 2008). Vacancy transfer probabilities from $K$ to $L$ for high atomic number elements at $123.6 \mathrm{keV}$ had been measured (Ertugral et al., 2005). K x-ray intensity ratios for Tantalum (Ta), Gold ( $\mathrm{Au})$, and Lead $(\mathrm{Pb})$ targets by 


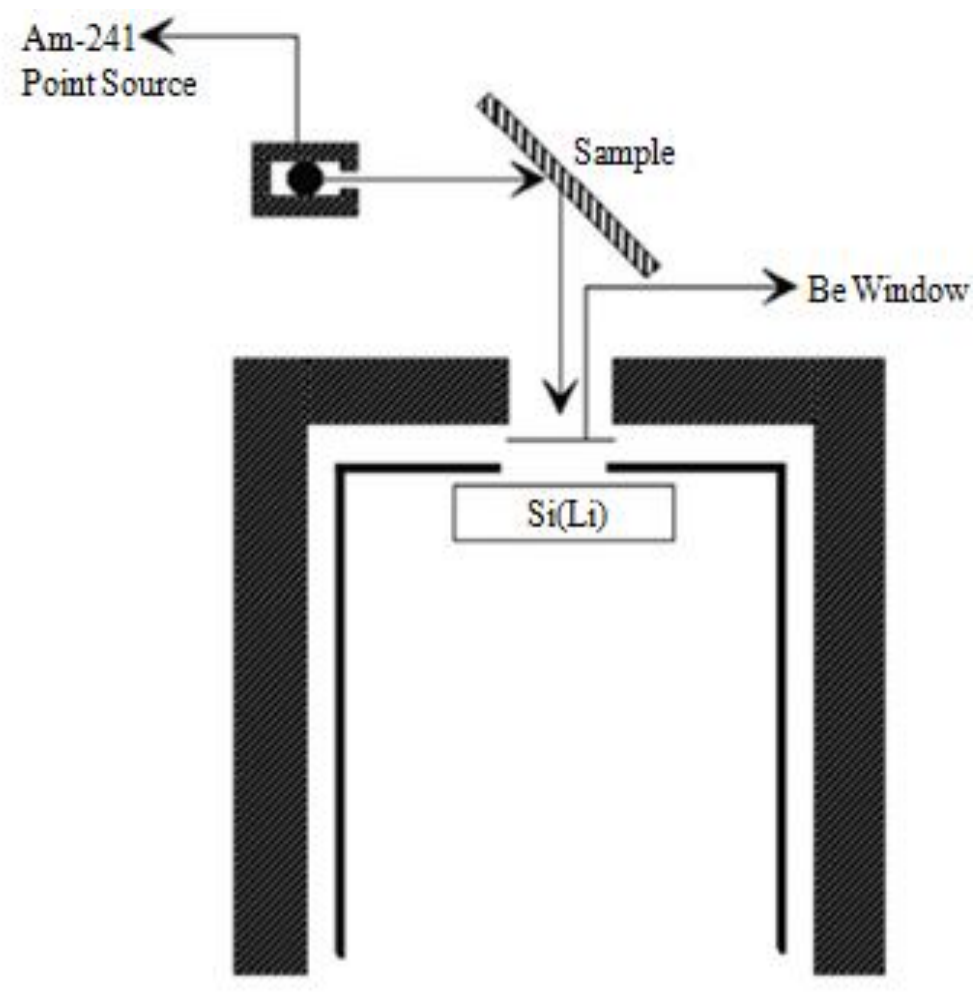

\section{Lead Shield}

Figure 1. Experimental setup.

adopting a $2 \pi$ geometrical configuration with weak radioactive ${ }^{57} \mathrm{Co}$ gamma source have been measured (Bennal and Badiger, 2006). Total vacancy transfer probabilities from $K$ to $L$ shell of selected elements in the atomic range $42 \leq Z \leq 82$ have been determined using weak ${ }^{57} \mathrm{Co}$ gamma source (Bennal et al., 2010). The average vacancy distributions have been calculated by researchers (Rao et al., 1972). $K$ to $L$ shell and $L$ to $M$ shell vacancy transfer probabilities for elements in the atomic range $37 \leq Z \leq 42$ and $18 \leq Z \leq 96$ have been measured, respectively (Puri et al., 1993). $K$ shell $x$-ray production cross-sections and fluorescence yields for Neodymium (Nd), Europium (Eu), Gadolinium (Gd), Dysprosium (Dy) and Holmium (Ho) have been measured using radioisotope $x$-ray fluorescence in the external magnetic field (Demir and Şahin, 2007).

In the present work, $K_{\beta} / K_{\alpha} \mathrm{x}$-ray intensity ratios, $\mathrm{K}$ shell fluorescence yields and radiative vacancy transfer probabilities from $K$ to $L$ shell for Zirconium ( $Z r$ ), Niobium $(\mathrm{Nb})$, Mo, Ruthenium (Ru), Rhodium (Rh), Pd, Silver $(\mathrm{Ag}), \mathrm{Cd}$, and $\mathrm{Sn}$ were investigated. The vacancies were produced by the $59.537 \mathrm{keV}$ photons from an ${ }^{241} \mathrm{Am}$ point source radioisotope. $K \mathrm{x}$-rays emitted by samples were detected using by a $\mathrm{Si}(\mathrm{Li})$ detector having a resolution of
$180 \mathrm{eV}$ full widths at half maximum at $5.9 \mathrm{keV}$.

\section{Experimental setup and method of measurement}

The experimental arrangement used for the present measurement shown in Figure 1. The parameters used for estimating $K_{\alpha, \beta}$ x-ray intensity ratios and total vacancy transfer probabilities are provided in Table 1. The samples were placed at a $45^{\circ}$ angle with respect to the beam from the source and excited by $59.537 \mathrm{keV}$ gamma rays from an ${ }^{241} \mathrm{Am}$ point source. The resultant fluorescent $\mathrm{x}$-rays were detected by a Si $(\mathrm{Li})$ detector (an active diameter $=6.2 \mathrm{~mm}$, sensitive crystal depth $=5 \mathrm{~mm}$, Be window thickness $=0.008 \mathrm{~mm}$, FWHM $=180 \mathrm{eV}$ at $5.9 \mathrm{keV}$ ) coupled to a analyzing system (Canberra-DSA1000). Spectroscopically high purity targets of Zirconium (Zr), Nb, Mo, Ru, Rh, Pd, Ag, Cd, and Sn foil samples of thickness ranging from $100 \mu \mathrm{m}$ to $1 \mathrm{~mm}$ and Ru (powder sample) have been used for the measurement. A typical $K \mathrm{x}$-ray spectrum of $\mathrm{Zr}$ is shown in Figure 2.

The $K_{a}$ x-ray production cross-section at excitation energy are given by:

$\sigma_{K_{\alpha}}=\sigma_{K} \omega_{K} f_{K_{\alpha}}$

Where, $\sigma_{K}$ is the total $K$ shell ionization cross-section, $w_{K}$ is the $K$ shell fluorescence yield and $f_{K \alpha}$ is given by: 
Table 1. Parameters used for estimating $K x$-ray intensity ratios and total vacancy transfer probabilities.

\begin{tabular}{lccccc}
\hline Sample & At. No & $\rho_{{ }}\left(\mathbf{g} / \mathbf{c m}^{2}\right)$ & $\sigma_{K}$ & $\beta_{K \alpha}$ & $\beta_{K \beta}$ \\
\hline Zirconium (Zr) & 40 & 0.163 & 463.77 & 0.9947 & 0.9946 \\
Niobium (Nb) & 41 & 0.214 & 528.29 & 0.9928 & 0.9927 \\
Molybdenum (Mo) & 42 & 0.334 & 557.76 & 0.9886 & 0.9885 \\
Ruthenium (Ru) & 44 & 0.126 & 663.73 & 0.9955 & 0.9954 \\
Rhodium (Rh) & 45 & 0.031 & 721.11 & 0.9988 & 0.9988 \\
Palladium (Pd) & 46 & 0.301 & 781.96 & 0.9889 & 0.9888 \\
Silver (Ag) & 47 & 0.105 & 845.96 & 0.9960 & 0.9959 \\
Cadmium (Cd) & 48 & 0.865 & 913.17 & 0.9677 & 0.9672 \\
Tin (Sn) & 50 & 0.552 & 1058.46 & 0.9786 & 0.9783 \\
\hline
\end{tabular}

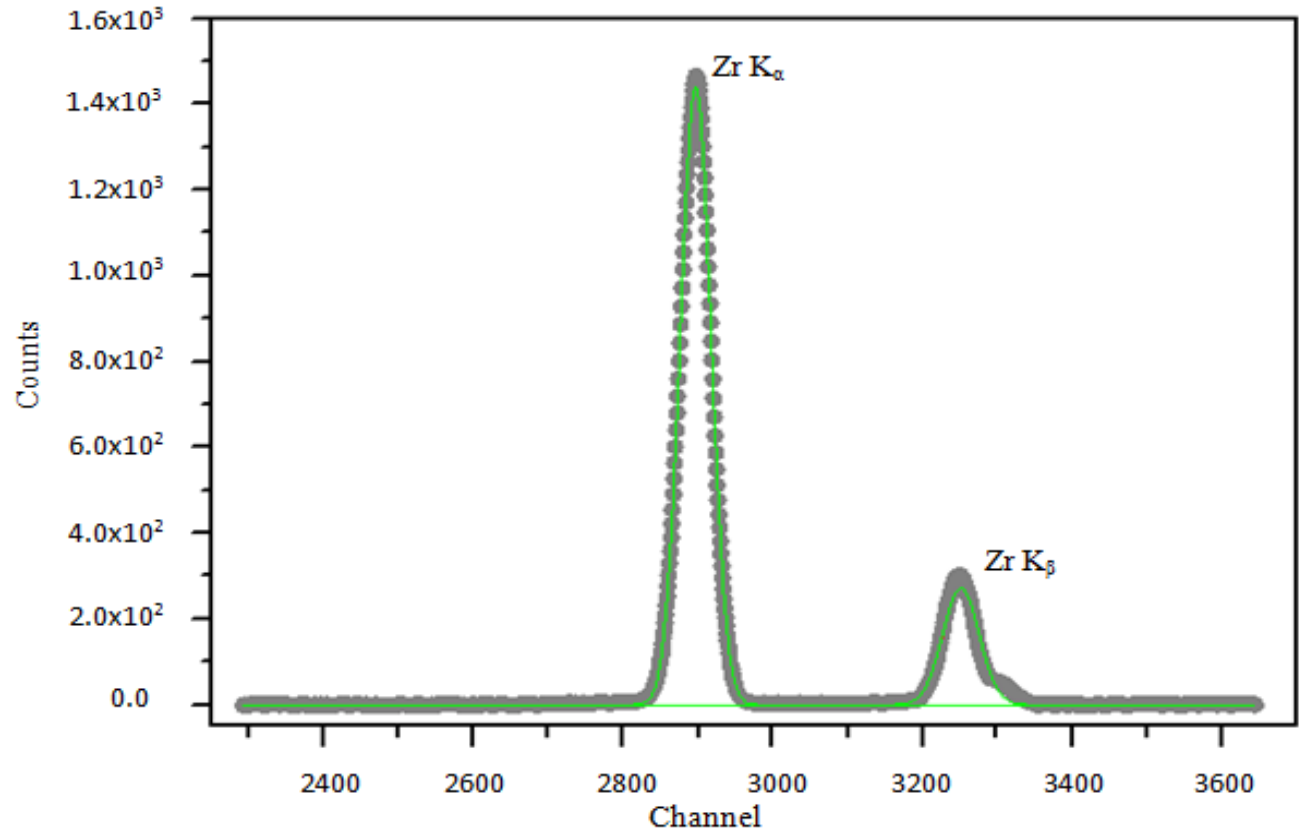

Figure 2. Typical $K \mathrm{x}$-ray spectrum for $Z$ r.

$f_{K a}=\left[1+I_{K_{\beta}} / I_{K_{\alpha}}\right]^{-1}$

The experimental $K_{\beta}$ to $K_{\alpha} \mathrm{x}$-ray intensity ratios are evaluated using the following equation:

$\frac{I_{K_{\beta}}}{I_{K_{\alpha}}}=\frac{N\left(K_{\beta}\right)}{N\left(K_{\alpha}\right)} \frac{\beta\left(K_{\alpha}\right)}{\beta\left(K_{\beta}\right)} \frac{\varepsilon\left(K_{\alpha}\right)}{\varepsilon\left(K_{\beta}\right)}$

Where, $N\left(K_{\alpha}\right)$ and $N\left(K_{\beta}\right)$ are net counts observed under the peaks corresponding $K_{\alpha}$ and $K_{\beta}$ x-rays, respectively, $\beta\left(K_{\alpha}\right)$ and $\beta\left(K_{\beta}\right)$ are the sample self-absorption corrections factors, and $\varepsilon\left(K_{\alpha}\right)$ and $\varepsilon\left(K_{\beta}\right)$ are photopeak efficiency values of the detector. The $\beta\left(K_{\alpha}\right)$ and $\beta\left(K_{\beta}\right)$ are the sample self-absorption corrections factors for the incident photon and emitted $K$ x-rays photons. The values of $\beta_{K}$ have been calculated by using the following expression obtained by assuming that the fluorescence $x$ rays are incident normally on the detector:

$\beta_{K}=\frac{1-\exp \left[-\left(\mu_{1} / \sin \theta+\mu_{2} / \sin \phi\right) t\right]}{\left(\mu_{1} / \sin \theta+\mu_{2} / \sin \phi\right) t}$

Where, $\mu_{1}$ and $\mu_{2}$ are the absorption coefficients, obtained by means of a computer program named WINXCOM (Gerward et al., 2004), at the incident and emitted x-rays photon energy. The product $I_{0} G \varepsilon$, containing the terms related to the incident photon flux, geometrical factor and the intrinsic absolute efficiency of the $x$ ray detector, was determined by collecting the $K_{\alpha}$ and $K_{\beta}$ x-ray spectra of samples for ${ }^{241} \mathrm{Am}$ in the same geometry using equation: 
Table 2. $K_{\beta} / K_{\alpha}$ x-ray intensity ratios.

\begin{tabular}{|c|c|c|c|}
\hline \multirow{2}{*}{ Sample } & \multicolumn{3}{|c|}{ Experiment } \\
\hline & $I_{K_{\beta}} / I_{K_{\alpha}}$ Present & Theoretical & Other \\
\hline $\mathrm{Zr}$ & $0.195 \pm 0.023$ & $\begin{array}{l}0.1913^{a} \\
0.1737^{b}\end{array}$ & $\begin{array}{c}0.1898 \pm 0.008^{c} \\
0.191 \pm 0.016^{d}\end{array}$ \\
\hline $\mathrm{Nb}$ & $0.197 \pm 0.022$ & $0.1772^{b}$ & $0.1993 \pm 0.008^{c}$ \\
\hline Mo & $0.201 \pm 0.022$ & $\begin{array}{l}0.1981^{a} \\
0.1809^{b}\end{array}$ & $\begin{array}{c}0.2016 \pm 0.004^{c} \\
0.193 \pm 0.014^{d}\end{array}$ \\
\hline $\mathrm{Ru}$ & $0.204 \pm 0.018$ & $0.1875^{b}$ & $0.198 \pm 0.016^{d}$ \\
\hline $\mathrm{Rh}$ & $0.207 \pm 0.018$ & $0.1906^{b}$ & $0.212 \pm 0.017^{d}$ \\
\hline $\mathrm{Pd}$ & $0.211 \pm 0.019$ & $0.1933^{b}$ & $0.207 \pm 0.014^{d}$ \\
\hline $\mathrm{Ag}$ & $0.214 \pm 0.022$ & $\begin{array}{l}0.1964^{\mathrm{a}} \\
0.1944^{\mathrm{b}}\end{array}$ & $\begin{array}{c}0.2096 \pm 0.004^{c} \\
0.217 \pm 0.015^{d}\end{array}$ \\
\hline $\mathrm{Cd}$ & $0.221 \pm 0.024$ & $0.1995^{b}$ & $\begin{array}{c}0.2035 \pm 0.006^{c} \\
0.217 \pm 0.015^{d}\end{array}$ \\
\hline Sn & $0.235 \pm 0.033$ & $\begin{array}{l}0.2230^{\mathrm{a}} \\
0.2061^{\mathrm{b}}\end{array}$ & $\begin{array}{c}0.2086 \pm 0.011^{c} \\
0.226 \pm 0.020^{d}\end{array}$ \\
\hline
\end{tabular}

Sources: a Scofield (1974a), ' Scofield (1974b), " Ertugral et al. (2007) and ${ }^{d}$ Ertugral et al. (2001).

$I_{0} G \varepsilon_{K_{i}}=\frac{N_{K_{i}}}{\sigma_{K_{i}} \beta_{K_{i}} m_{i}} \quad(i=\alpha, \beta)$

Where, $N_{K_{i}}$ is the measured intensity, $I_{0}$ intensity of incident radiation, $G$ a geometrical factor, $\varepsilon_{K_{i}}$ detection efficiency, $m_{i}$ and the thickness of target in $\mathrm{g} / \mathrm{cm}^{2}$ and $\beta_{K_{i}}$ is the self- absorption correction for the target material.

The experimental $K$ to $L$ shell total vacancy transfer probabilities, $\eta_{K L}$, were calculated by using following equation:

$\eta_{K L}=\frac{2-\omega_{K}}{1+\left(I_{K \beta} / I_{K \alpha}\right)}$

Where, $\omega_{K}$ is the fluorescence yield and $I_{K \beta} / I_{K \alpha}$ is the intensity ratio of the $K$ x-rays. The quantities $\eta_{K L_{i}}$ are defined as the averaged number of primary $L_{i}$ subshell vacancies produced by one vacancy in the $K$ shell through radiative $\eta_{K L_{i}}(\mathrm{R})$ and nonradiative $\eta_{K L_{i}}(\mathrm{~A})$ transitions:

$\eta_{K L_{i}}=\eta_{K L_{i}}(\mathrm{R})+\eta_{K L_{i}}(\mathrm{~A})$
The quantity $\eta_{K L_{i}}(\mathrm{R})$ is proportional to probability that a $K-L_{i}$ radiative transition takes place

$\eta_{K L_{i}}(\mathrm{R})=w_{K}\left[\frac{I\left(K L_{i}\right)}{I_{K}(R)}\right]$

Where, $I\left(K L_{i}\right)$ is the $K-L_{i}$ x-ray intensity and $I_{K}(R)$ is the total intensity of $K$ x-rays. The $I\left(K L_{1}\right)$ intensity is negligible because $K-L_{1}$ transitions are forbidden according to the selection rule $\Delta l=0, \pm 1, l+l^{\prime} \geq 1$ ( $l$ is the orbital angular momentum quantum number) for radiative transitions. Theoretical $\eta_{K L_{i}}(R)$ radiative vacancy transfer probabilities were calculated using the equation:

$\eta_{K L_{i}}(R)=\frac{\Gamma_{R}\left(K L_{i}\right)}{\Gamma_{K}}$

\section{RESULTS AND DISCUSSION}

The measured values of the $K_{\beta} / K_{\alpha} x$-ray intensity ratios in the atomic range $40 \leq Z \leq 50$ are listed in Table 2 with the 
Table 3. Kx-ray fluorescence yields.

\begin{tabular}{|c|c|c|c|}
\hline \multirow{2}{*}{ Sample } & \multicolumn{3}{|c|}{ Experiment } \\
\hline & $w_{K}$ present & Theoretical & Other \\
\hline \multirow{2}{*}{$\mathrm{Zr}$} & \multirow{2}{*}{$0.729 \pm 0.037$} & $0.730^{\mathrm{a}}$ & \multirow[t]{2}{*}{$0.745 \pm 0.060$} \\
\hline & & $0.734^{b}$ & \\
\hline \multirow{2}{*}{$\mathrm{Nb}$} & \multirow{2}{*}{$0.747 \pm 0.038$} & $0.747^{\mathrm{a}}$ & \multirow[t]{2}{*}{$0.747 \pm 0.060^{\circ}$} \\
\hline & & $0.751^{b}$ & \\
\hline \multirow{2}{*}{ Mo } & \multirow{2}{*}{$0.765 \pm 0.039$} & $0.765^{\mathrm{a}}$ & \multirow[t]{2}{*}{$0.769 \pm 0.011^{\circ}$} \\
\hline & & $0.767^{b}$ & \\
\hline $\mathrm{Ru}$ & $0.749 \pm 0.038$ & $0.749^{\mathrm{a}}$ & - \\
\hline \multirow{2}{*}{$\mathrm{Rh}$} & \multirow{2}{*}{$0.808 \pm 0.042$} & $0.808^{\mathrm{a}}$ & \multirow[t]{2}{*}{ - } \\
\hline & & $0.809^{b}$ & \\
\hline \multirow{2}{*}{$\mathrm{Pd}$} & \multirow{2}{*}{$0.820 \pm 0.042$} & $0.820^{a}$ & \multirow[t]{2}{*}{-} \\
\hline & & $0.820^{\mathrm{b}}$ & \\
\hline \multirow{2}{*}{$\mathrm{Ag}$} & \multirow{2}{*}{$0.831 \pm 0.043$} & $0.831^{\mathrm{a}}$ & \multirow[t]{2}{*}{$0.834 \pm 0.010^{\circ}$} \\
\hline & & $0.831^{b}$ & \\
\hline \multirow{2}{*}{$\mathrm{Cd}$} & \multirow{2}{*}{$0.843 \pm 0.043$} & $0.843^{\mathrm{a}}$ & \multirow[t]{2}{*}{$0.839 \pm 0.067^{\circ}$} \\
\hline & & $0.842^{b}$ & \\
\hline \multirow{2}{*}{ Sn } & \multirow{2}{*}{$0.862 \pm 0.044$} & $0.862^{\mathrm{a}}$ & \multirow[t]{2}{*}{$0.858 \pm 0.069^{\circ}$} \\
\hline & & $0.860^{\mathrm{b}}$ & \\
\hline
\end{tabular}

Sources: ${ }^{a}$ Krause (1979), ${ }^{b} \mathrm{Hubbell}$ (1989), ${ }^{\mathrm{C}} \mathrm{Han}$ et al. (2007) and ${ }^{\mathrm{d}} \mathrm{Bennal}$ et al. (2010).

theoretical and other experimental values. Theoretical values are based on relativistic Hartee-Fock and HartreeSlater theories (Scofield, 1974a, b). As seen from Table 2 , the agreement between the present results and theoretical prediction of Scofield is within the range 3\%. From Table 2, our measured values closely agree with the values obtained from other researchers (Ertuğrul et al., 2001). The overall error in the experimental parameters is the sum of the uncertainties in different factors, namely, the evaluation of peak areas (2.25 to $6.20 \%$ ), target mass thickness (1.65 to $4.60 \%$ ) and statistical error $(<1.00 \%)$. Total errors affecting the experimental parameters are calculated between 2.96 to $7.78 \%$. The errors in the evaluation of the areas under the $X$ - and gamma-ray peaks have two main error sources, that is., the errors in the elimination of the background and in the peak fitting procedures.

The measured values of the $K$ shell fluorescence yield $w_{K}$ for elements in the atomic range $40 \leq Z \leq 50$ are compared with the theoretical values (Krause, 1979; Hubbell, 1989) and other experimental values (Han et al., 2007; Bennal et al., 2010) in Tables 3. As seen from
Table 3, the measured $w_{K}$ values increase with increasing atomic number. Also, our experimental values closely agree with the calculated values and other experimental results.

In Table 4, we present our values of $K$ to $L$ shell total vacancy transfer probabilities for the elements in the atomic range $40 \leq Z \leq 50$, together with theoretical and other experimental values. The error in $\eta_{K L}$ values is less than $7.1 \%$ for all targets and it mainly comes from the uncertainty in $w_{K}$ and $K_{\beta} / K_{\mathrm{a}}$. As seen from Table 4 , our measured values of $\eta_{K L}$ agree with theoretical and other experimental values. We believe that the present experimental values may serve three purposes. Firstly, our values confirm the reliability of the existing theoretical values. Secondly, with the vacancy transfer probability values that we have obtained in this study, the elemental analysis of different samples can be calculated with confidence. Thirdly, the experimental values of these vacancy transfer probabilities can be used to calculate the experimental absorption jump factors and jump ratios of $\mathrm{K}$ shell, $\mathrm{L}$ shell-subshell. In addition, the agreement 
Table 4. $K-L$ total vacancy transfer probabilities.

\begin{tabular}{cccc}
\hline \multirow{2}{*}{ Sample } & \multicolumn{3}{c}{ Experiment } \\
\cline { 2 - 4 } & $\eta_{K L}$ present & Theoretical & Other \\
\hline $\mathrm{Zr}$ & $1.063 \pm 0.0011$ & $1.064^{\mathrm{d}}$ & $1.061 \pm 0.07^{\mathrm{c}}$ \\
$\mathrm{Nb}$ & $1.047 \pm 0.0002$ & $1.049^{\mathrm{d}}$ & $1.044 \pm 0.06^{\mathrm{c}}$ \\
$\mathrm{Mo}$ & $1.028 \pm 0.0001$ & & $1.039 \pm 0.005^{\mathrm{a}}$ \\
$\mathrm{Ru}$ & $1.039 \pm 0.0004$ & $1.030^{\mathrm{d}}$ & $1.03 \pm 0.08^{\mathrm{b}}$ \\
$\mathrm{Rh}$ & $0.988 \pm 0.0001$ & & - \\
$\mathrm{Pd}$ & $0.974 \pm 0.0001$ & $1.000^{\mathrm{d}}$ & - \\
$\mathrm{Ag}$ & $0.962 \pm 0.0002$ & $0.984^{\mathrm{d}}$ & $0.99 \pm 0.08^{\mathrm{b}}$ \\
$\mathrm{Cd}$ & $0.947 \pm 0.0006$ & $0.963^{\mathrm{d}}$ & \\
& & $0.958^{\mathrm{d}}$ & $0.973 \pm 0.006^{\mathrm{a}}$ \\
$\mathrm{Sn}$ & $0.925 \pm 0.0008$ & & $0.952^{\mathrm{d}}$ \\
\hline
\end{tabular}

${ }^{a}$ Bennal et al. (2010), ${ }^{b}$ Santra et al. (2005), ${ }^{c} E . O ̈ z ~(2006), ~{ }^{d}$ Rao et al. (1972).

between experimental and theoretical values indicates that the usage of XRF technique is beneficial for these studies.

\section{REFERENCES}

Bennal AS, Badiger NM (2006). Measurement of $K-L$ radiative vacancy transfer probabilities for $\mathrm{Ta}, \mathrm{Au}$ and $\mathrm{Pb}$ in a $2 \pi$ geometrical configuration. Nucl. Inst. Meth. B. 247:161-165.

Bennal AS, Niranjan KM, Badiger NM (2010). Determination of K to $L$ shell total vacancy transfer probabilities using a weak gamma source: An alternative method. J. Quant. Spect. Rad. Tra. 111:1363-1371.

Demir D, Şahin Y (2007). Measurement of $K$-shell $x$-ray production cross section and $K$ to $L$ and $M$-shell radiative vacancy transfer probabilities for $\mathrm{Nd}$, Eu, Gd, Dy and Ho at excitation with $59.5 \mathrm{keV}$ photons in external magnetic field. X-Ray Spectrom. 36:178-184.

Ertugral B, Apaydın G, Baltaş H, Çevik U, Ertuğrul M, Kobya Ail (2007). $\mathrm{Kb} / \mathrm{Ka} \mathrm{X}$-ray intensity ratios for elements in the range $16 \leq Z \leq 92$ excited by $5.9,59.5$ and $123.6 \mathrm{keV}$ photons. Radiat. Phys. Chem. 76:15-22.

Ertugral B, Apaydin G, Baltas H, Çevik U, Kobya Al, Ertugrul M (2005). Measurement of vacancy transfer probabilities from $K$ to $L$ shell for high atomic number elements. Spectrochimica Acta B. 60:519-524.

Ertugral M, Söğüt Ö, Şimşek Ö, Büyükkasap E (2001). Measurement of $\mathrm{Kb} / \mathrm{Ka}$ intensity ratios for elements in the range $22 \leq \mathrm{Z} \leq 69$ at $59.5 \mathrm{keV}$. J. Phys. B. 34:909-914.

Ewart P (2008). X-Rays: Transitions involving inner shell electrons, Atomic Physics, University of Oxford, Oxford. P. 56.

Gerward L, Guilbert N, Jensen KB, Levring H (2004). WinXCom-a program for calculating X-ray attenuation coefficients. Rad. Phys. Chem. 71:653-654.
Han I, Şahin M, Demir L, Şahin Y (2007). Measurement of $K$ X-ray fluorescence cross-sections, fluorescence yields and intensity ratios for some elements in the atomic range $22 \leq Z \leq 68$. Appl. Radiat. Isot. 65:669-675.

Hubbell JH (1989). Bibliography and Current Status of $K, L$ and Higher Shell Fluorescence Yields for Computations of Photon EnergyAbsorption Coefficients. NISTIR 89-4144.

Krause MO (1979). Atomic radiative and radiationless yields for $K$ and $L$ shell. J. Phys. Chem. Ref. Data. 8:307-327.

Oz E (2006). Determination of ratios of emission probabilities of Auger electrons and $K-L$-shell radiative vacancy transfer probabilities for 17 elements from Mn to Mo at $59.5 \mathrm{keV}$, J. Quant. Spectrosc. Radiat. Transf. 97:41-50.

Puri S, Mehta D, Chand B, Nirmal S, Trehan PN (1993). Measurements of $K$ to $L$ shell vacancy transfer probability for the elements $37 \leq Z \leq$ 42 Nucl. Instr. Meth. B. 73:443-446.

Rao PV, Chen MH, Crasemann B (1972). Atomic Vacancy Distributions Produced by Inner-Shell Ionization. Phys. Rev. A.5:997-1012.

Rao PV, Chen MH, Crasemann B (1972). Atomic vacancy distributions produced by inner-shell ionization. Phys. Rev. A. 5:997-1011.

Santra S, Mandal AC, Mitra D, Sarkar M, Bhatacharya D (2005). Measurement of the $K$ to $L$ shell vacancy transfer probabilities for Mo, $\mathrm{Pd}$ and $\mathrm{Cd}$ in photon impact. Radiat. Phys. Chem. 74:282-287.

Scofield JH (1974a). Exchange corrections of KX-ray emission rates. Phys. Rev. A. 9:1041-1047.

Scofield JH (1974b). Relativistic Hartree-Slater values for K and L X-ray emission rates. At. Data Nucl. Data Tables. 14:121-137.

Tuzluca F, Söğüt Ö, Büyükkasap E, Durdu BG, Küçükönder A (2008). Measurements of radiative vacancy transfer probabilities from $L_{3}$ subshells to $M, N$ and $O$ shells and subshells in the atomic range 72 $\leq Z \leq 92$. Radiat. Phys. Chem. 77:996-1001. 